\title{
Marginal and internal fit of zirconia copings obtained using different digital scanning methods
}

\section{Lorena Oliveira PEDROCHE(a) Sérgio Rocha BERNARDES(b) Moira Pedroso LEÃO(a) Cibele Cândida de Almeida KINTOPP(a) \\ Gisele Maria CORRER(a) \\ Bárbara Pick ORNAGHI(a) \\ Carla Castiglia GONZAGA(a)}

(a) Universidade Positivo, Graduate Program in Dentistry, Curitiba, PR, Brazil.

(b)|nstituto Latino Americano de Pesquisa e Ensino - ILAPEO, Curitiba, PR, Brazil.

Declaration of Interests: The authors certify that they have no commercial or associative interest that represents a conflict of interest in connection with the manuscript.

Corresponding Author:

Carla Castiglia Gonzaga

E-mail: carlacgonzaga2@gmail.com

DOI: 10.1590/1807-3107BOR-2016.vol30.0113

Submitted: Nov 12, 2015

Accepted for publication: Jun 29, 2016

Last revision: Aug 10, 2016
Abstract: The objective of this study was to evaluate the marginal and internal fit of zirconia copings obtained with different digital scanning methods. A human mandibular first molar was set in a typodont with its adjacent and antagonist teeth and prepared for an all-ceramic crown. Digital impressions were made using an intraoral scanner (3Shape). Polyvinyl siloxane impressions and Type IV gypsum models were also obtained and scanned with a benchtop laboratory scanner (3Shape D700). Ten zirconia copings were fabricated for each group using CAD-CAM technology. The marginal and internal fit of the zirconia copings was assessed by the silicone replica technique. Four sections of each replica were obtained, and each section was evaluated at four points: marginal gap (MG), axial wall (AW), axio-occlusal edge (AO) and centro-occlusal wall (CO), using an image analyzing software. The data were submitted to one-way ANOVA and Tukey's test $(\alpha=0.05)$. They showed statistically significant differences for MG, AO and CO. Regarding MG, intraoral scanning showed lower gap values, whereas gypsum model scanning showed higher gap values. Regarding AO and $\mathrm{CO}$, intraoral digital scanning showed lower gap values. Polyvinyl siloxane impression scanning and gypsum model scanning showed higher gap values and were statistically similar. It can be concluded that intraoral digital scanning provided a lower mean gap value, in comparison with conventional impressions and gypsum casts scanned with a standard benchtop laboratory scanner.

Keywords: Dental Marginal Adaptation; Zirconium Oxide; Computer-Aided Design.

\section{Introduction}

Clinical studies have estimated that from $2 \%$ to $6 \%$ of restorations with yttrium-stabilized zirconia copings fail after 3 to 5 years due to secondary caries, loss of retention, and ceramic veneer chipping ${ }^{1,2}$. The two first-mentioned causes may occur because of dissolution of the luting cement, which is a consequence of deficiencies in marginal adaptation of the prosthetic restoration ${ }^{3}$.

The processing of zirconia for the fabrication of single and multiple fixed partial restorations was made possible only after advanced improvements in CAD-CAM technology, which is based on a series of processing steps, such as scanning, software designing of the restoration, milling and sintering. 
All these steps may interfere individually or jointly with the precision of fit of indirect restorations $s^{4,5}$. The most critical step in the fabrication of zirconia fixed prostheses is obtaining an accurate impression of the preparation, which can be done using conventional or digital methods. Conventional impression has several known disadvantages, such as patient discomfort, distortion of the impression and die materials, imprecision in detail reproduction, and bacterial contamination, which requires disinfection of the impressions before casting the working models ${ }^{5,6,7,8,9}$.

In contrast, digital impression techniques have eliminated such conventional impression drawbacks. However, it should be made clear that digital scanning methods include the use of intraoral digital scanners or benchtop laboratory scanners for polyvinyl siloxane impressions and gypsum models. When laboratory scanners are used, conventional impression is still needed; in comparison, intraoral scanners are more widely used in clinical dental practice, where their application definitively simplifies the workflow and avoids inaccuracies related to conventional impression techniques and model casting ${ }^{10,11}$.

Nevertheless, not all the aspects related to intraoral digital scanning may be considered favorable, since the equipment requires a relatively high initial investment, and prospective users must learn how to use it and adapt this system to the dental office routine $^{10}$. Moreover, intraoral digital scanners have limitations in some clinical situations. For example, the scanning procedure is complicated when finish lines are located subgingivally, and is worsened by the presence of blood or saliva, and also when used in the molar region, because of the limited space in the oral cavity, making access of the scanning wand difficul $t^{11}$. Moreover, some intraoral scanners require the application of a powder to eliminate the superficial brightness, and may present some dimensional error due to camera misalignment and low accuracy of the optical equipment ${ }^{12,13}$.

Recent studies have indicated that the marginal and internal fit is similar for indirect restorations obtained using both conventional and intraoral digital impression methods $\mathrm{s}^{14,15,16}$. However, little evidence is available with regard to the accuracy of fit provided by intraoral digital impression scanners, in comparison with laboratory scanners, when used to acquire images either for polyvinyl siloxane impressions or gypsum models. Thus, the aim of this study was to evaluate the marginal and internal fit of zirconia copings obtained using an intraoral scanning system and benchtop laboratory scanning system, in which both the polyvinyl siloxane impression and the gypsum model were scanned. The hypothesis tested was that there was no difference in the marginal and internal fit of zirconia copings, irrespective of the scanning method used.

\section{Methodology}

A human mandibular first molar was set in a typodont and prepared for an all-ceramic crown with a medium-grit diamond bur (4138, KG Sorensen, Cotia, Brazil). The prepared tooth had the following features: supragingival circumferential chamfer finish line, $2.0 \mathrm{~mm}$ occlusal reduction, $1.5 \mathrm{~mm}$ axial reduction, axial convergence angle of $6^{\circ}$ and rounded angles. A silicone putty matrix of the tooth was obtained and used to help control the occlusal and axial reductions. The angulation was controlled by keeping the head of the diamond bur parallel to the long axis of the preparation.

Three different digital scanning methods were performed $(n=10)$ : a. intraoral digital scanning (direct digitalization); b. scanning of polyvinyl siloxane impressions (indirect digitalization); c. scanning of the gypsum cast/models with a laboratory digital scanner (indirect digitalization).

The procedure to assess the intraoral digital scanning group involved digitally scanning the maxillary and mandibular arches with an intraoral scanner (Trios 3Shape, Copenhagen, Demark), after which another scan was performed to register the occlusion.

The second group was assessed by obtaining single-step full-arch polyvinyl siloxane impressions (HydroXtreme, Coltene Whaledent, Altstätten, Switzerland). The heavy-body material was loaded into a stock tray and the light-body silicone was placed over the prepared tooth. The loaded tray was seated firmly on the mandibular arch and allowed to set for 5 minutes. A maxillary impression was also made for each mandibular arch impression with heavy-body 
polyvinyl siloxane. Each pair of polyvinyl siloxane impressions was sprayed with surface disinfectant, and rinsed. The impressions were then digitally scanned with a benchtop laboratory scanner (3Shape D700, Copenhagen, Denmark). A non-aqueous developer (SKD-S2, Spotcheck, Embu das Artes, Brazil) was applied to the impression surfaces to reduce brightness and improve reproduction.

For the third group, single-step full-arch polyvinyl siloxane impressions (HydroXtreme, Coltene Whaledent, Altstätten, Switzerland) were obtained and disinfected as described above. After a waiting period of 30 minutes, each impression was cast in Type IV dental stone (Durone IV, Dentsply, York, USA) by manual manipulation, following the manufacturer's instructions. The resulting models were then scanned with the same benchtop laboratory scanner.

All the digital scans were used to design zirconia copings (Dental Designer Premium 2013 software, 3Shape, Copenhagen, Denmark) by the same experienced dental technician. One zirconia coping (Metoxit, Thayngen, Switzerland) was fabricated per scan. All copings were produced in a milling center (Neodent, Curitiba, Brazil), using a five-axis grinding machine (Ultrasonic 20 linear, DMG Mori Aktiengesellschaft, Bielefeld, Germany), which considered a marginal gap of $10 \mu \mathrm{m}$, and an internal gap of $50 \mu \mathrm{m}$ for the axial and occlusal walls, and $70 \mu \mathrm{m}$ for the axio-occlusal angle. After milling, the zirconia copings were sintered according to the manufacturer's recommendations.

The marginal and internal fit of the copings was assessed by the silicone replica technique $e^{11,17,18,19}$, using a light-body and a regular-body polyvinyl siloxane (HydroXtreme, Coltene Whaledent, Altstätten, Switzerland). The replica was sectioned into four pieces using a sharp scalpel blade. Two cuts were made in each silicone replica, one buccolingually and another mesiodistally.

The four cross sections of each replica were placed on a bench scanner (C3180 HP Photosmart, HP, Palo Alto, USA) for digitization. The high-resolution images (1,200 dpi) were saved as jpg files and a single operator determined the thickness of the light-body polyvinyl siloxane using an image analyzing software (ImageJ, U. S. National Institutes of Health, Bethesda, USA).
The thickness of each fragment of the replica was measured at four points, as described below and shown in Figure 1:

a. marginal gap (MG): perpendicular distance from the internal surface at the margin of the coping to the prepared tooth;

b. axial wall (AW): discrepancy between the prepared tooth and internal surface of the coping at the mid-axial wall;

c. axial-occlusal angle (AO): discrepancy between the prepared tooth and internal surface of the coping in the region of the axial-occlusal edge, at the point of intersection of two straight lines: one parallel to the occlusal plane and the other parallel to the axial wall;

d. centro-occlusal area (CO): discrepancy between the prepared tooth and internal surface of the coping in the center of the occlusal region.

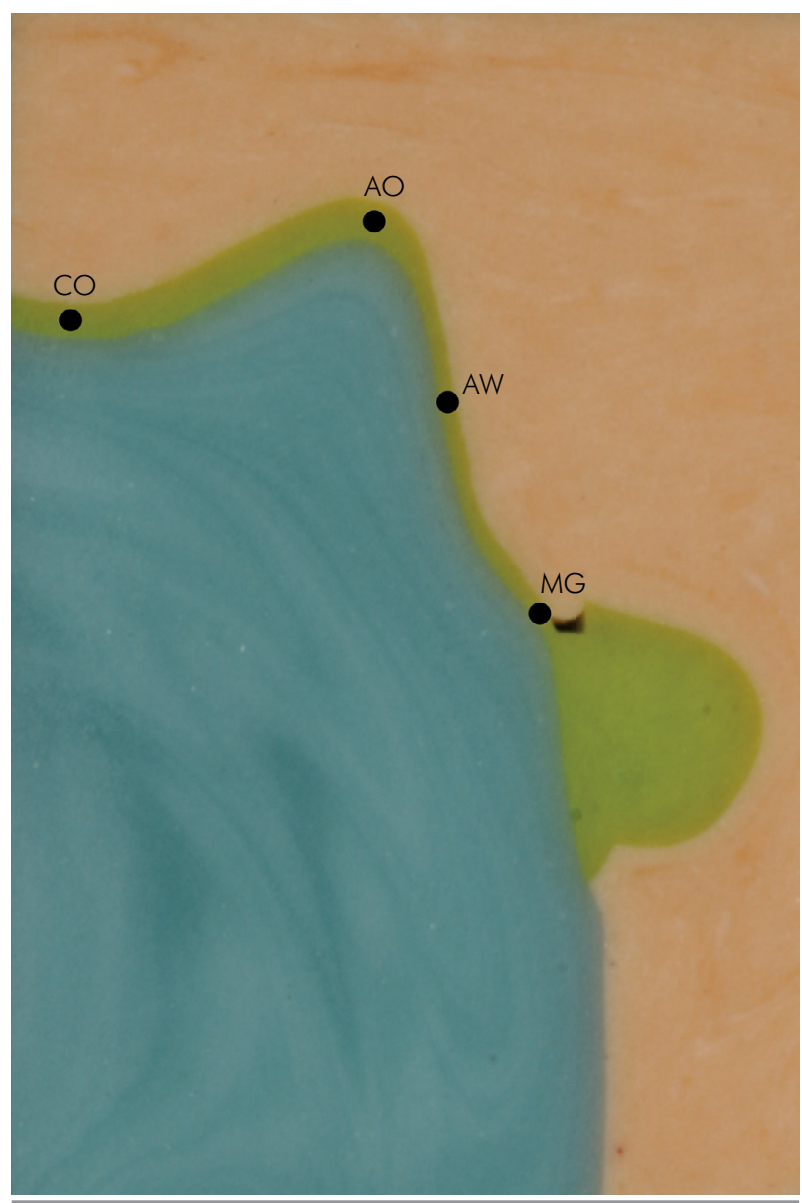

Figure 1. Replica cross section indicating the location of the four measurement points. 
Therefore, 16 measurements were obtained for each replica, i.e. four measurements for each point. The mean for each point was calculated and considered for statistical analysis. The normality of the data was checked using the Shapiro-Wilk test and the homoscedasticity was tested using Levene's test for homogeneity of variances. The data were submitted to one-way ANOVA for each point of measurement and Tukey's test, both with a significance level of 0.05 .

\section{Results}

The results for the normality and the homogeneity of variance tests, for each point of measurement, were, respectively: $\mathrm{MG}(\mathrm{p}=0.061$ and 0.155$), \mathrm{AW}(\mathrm{p}=0.462$ and 0.489), $\mathrm{AO}(\mathrm{p}=0.314$ and 0.220$)$ and $\mathrm{CO}(\mathrm{p}=0.048$ and 0.074).

The means and standard deviations for all the groups and measurement points are shown in Table 1. The data showed statistically significant differences based on the digital scanning methods for MG, $\mathrm{AO}$ and $\mathrm{CO}$. There was no statistically significant difference among the gap values for AW. Regarding MG, intraoral digital scanning showed lower gap values and gypsum model scanning showed higher gap values. Polyvinyl siloxane impression scanning showed intermediate misfit values. Regarding $\mathrm{AO}$ and $\mathrm{CO}$, intraoral digital scanning also showed lower gap values. Polyvinyl siloxane impression scanning and gypsum model scanning showed higher gap values and were statistically similar.

\section{Discussion}

Previous studies have demonstrated that using intraoral digital impression for the fabrication of ceramic fixed prostheses has resulted in significantly smaller marginal gaps than using conventional impression methods ${ }^{14,19}$. The results of the present study corroborate this finding, considering that the intraoral digital scanning group largely presented the lowest mean misfit value, compared with the groups in which laboratory digital scanning methods were used. This may be explained by the fact that there was no need for impression or casting materials to perform intraoral scanning. These materials, irrespective of their type and quality, undergo some degree of dimensional change $\mathrm{e}^{20,21}$. When intraoral digital scanning is used for fabrication of structures of single (copings or crowns) and multiple fixed partial restorations, the impression and cast steps may be eliminated, thus contributing to obtaining a more precise dental prosthesis ${ }^{19}$.

In the present study, the replica technique was used to evaluate the marginal and internal adaptation of zirconia copings. This technique is well described in the literature and is established as a non-invasive and reliable method to determine the marginal and internal gap of indirect restorations, since it is easy and not time-consuming to perform, and relatively inexpensive $e^{17,19,22,23}$.

The precision of fit of indirect restorations is related to a minimal marginal gap between the prepared tooth and the prosthesis. In order to ensure clinical longevity, a marginal gap of no more than $120 \mu \mathrm{m}$ is considered clinically acceptable ${ }^{24}$. The importance of marginal fit lies in the fact that the major causes of indirect restoration failure are secondary caries and loss of retention, factors closely related to the dissolution of the luting cement and deficiencies in marginal adaptation. As important as marginal fit, a minimum and uniform internal gap is also a desirable aspect of indirect restorations, since large

Table 1. Means and standard deviations for the misfit values of the three scanning methods and four points of measurement.

\begin{tabular}{|c|c|c|c|c|}
\hline \multirow{2}{*}{ Scanning method } & \multicolumn{4}{|c|}{ Measurement points $(\mu \mathrm{m})$} \\
\hline & MG & AW & $\mathrm{AO}$ & $\mathrm{CO}$ \\
\hline Gypsum model scanning & $87.0 \pm 31.0^{a}$ & $40.2 \pm 8.5^{a}$ & $373.8 \pm 86.0^{a}$ & $300.9 \pm 95.6^{\circ}$ \\
\hline Polyvinyl siloxane impression scanning & $71.1 \pm 19.1 \mathrm{ab}$ & $36.2 \pm 9.0^{\circ}$ & $340.8 \pm 60.4^{a}$ & $248.4 \pm 77.6^{\circ}$ \\
\hline Intraoral digital scanning & $59.2 \pm 14.3^{b}$ & $40.0 \pm 6.6^{a}$ & $182.0 \pm 32.7^{b}$ & $112.6 \pm 59.3^{b}$ \\
\hline p value & 0.035 & 0.474 & $<0.001$ & $<0.001$ \\
\hline
\end{tabular}

Values followed by the same superscript in the columns are statistically similar ( $p>0.05$ ); MG: marginal gap; AW: axial wall; AO: axial-occlusal angle; $\mathrm{CO}$ : centro-occlusal area. 
and inhomogeneous internal gaps may adversely affect the retention or the resistance of the restoration ${ }^{25}$.

Accordingly, it was important to compare the mean gap values among the points of measurement, according to the scanning method used. Although there was no difference among the three scanning methods for AW, digital intraoral scanning of MG, $\mathrm{AO}$ and $\mathrm{CO}$ resulted in statistically lower gap values than the laboratory scanning methods. These results are further corroborated by the fact that the intraoral digital scanning provides better adaptation for copings than impression or cast scanning.

In the present study, the gap for MG and AW means ranged from 36.2 to $87.0 \mu \mathrm{m}$, both values being within the limit of marginal misfit considered clinically acceptable. Nevertheless, when analyzing the misfit values obtained for $\mathrm{AO}$ and $\mathrm{CO}$, it can be observed that the values ranged from 112.6 to $373.8 \mu \mathrm{m}$, irrespective of the digital scanning method evaluated. Some of these values may be above the clinically acceptable value, but they were similar to those obtained in other laboratory and clinical studies s,26,27. $^{25}$.

The increasing discrepancies at $\mathrm{AO}$ and $\mathrm{CO}$ can be attributed to the geometry of the milling burs, since the diameter of the bur defines the smallest grindable radius of the internal surface of the coping ${ }^{27}$. Another possible reason is a phenomenon called "overshooters," which produce virtual peaks near the edges of three-dimensional structures when captured on digital scanning, and which may cause distortion of the prepared tooth design in angle regions ${ }^{28}$.

The present study used three different scanning protocols: one intraoral direct scanning, and two indirect scannings for impressions and models. In so doing, the data were captured from surfaces with different optical characteristics, including translucency, reflection, and smoothness. The intraoral and laboratory scanners may not have captured the data with the same degree of accuracy. In some circumstances, accurate capture of the image calls for the surface to be coated with an opaque spray or dye. In the present study, the impressions were coated with a non-aqueous developer to reduce brightness and improve the readings ${ }^{25}$. In another in vivo study, the precision of intra- and extraoral (direct and indirect) digital scanners was evaluated ${ }^{29}$. The authors concluded that the intraoral scanner was less precise, because of the conditions of the oral cavity, such as the presence of saliva and limited access by the scanner. This result should be taken into consideration when analyzing our results, because this was a laboratory study that was not subject to the influence of such factors.

Therefore, when analyzing the results of the present study, the tested hypothesis was accepted irrespective of the point of measurement, because intraoral scanning provided lower overall mean misfit values, in comparison with the indirect scanning methods.

Since the literature points out that a marginal gap of no more than $120 \mu \mathrm{m}$ is considered clinically acceptable to ensure the clinical longevity of a restoration, this value was considered a reference for the present study. Metal copings made by the lost wax technique were not included as controls, because this study aimed to compare the marginal and internal fit of zirconia copings obtained using different digital scanning methods. This considered, the inclusion of metal copings fabricated with the lost wax technique to be used as controls would add more variables than needed to achieve the study objectives (two different materials - zirconia versus metal - and coping fabrication methods - milling versus lost wax technique). Therefore, scanning of the gypsum cast/models may be considered as the control group, since it is the most common method for fabrication of zirconia copings.

Moreover, it should be considered that the majority of dentists feel comfortable using conventional impression-taking materials and techniques for the fabrication of single crowns and fixed dental prostheses. With this in mind, it is important to point out that it may be difficult to change from a successful technique of moderate cost to a less widely known technique of high cost. Therefore, considering the cost-benefit of intraoral digital scanning, one must ponder whether the advantages of the intraoral digital scanning technique outweigh the familiarity and clinical success obtained with conventional impression procedures ${ }^{10}$.

\section{Conclusion}

Based on the results of the present research, it was concluded that intraoral digital scanning 
provided a lower mean gap value for three of the four measurement points, in comparison with conventional impressions and gypsum casts scanned with a standard benchtop laboratory scanner.

\section{References}

1. Della Bona A, Kelly JR. The clinical success of all-ceramic restorations. J Am Dent Assoc. 2008;139 Suppl:8S-13S. doi: 10.14219/jada.archive.2008.0361

2. Suárez MJ, Lozano JF, Paz Salido M, Martínez F. Three-year clinical evaluation of In-Ceram Zirconia posterior FPDs. Int J Prosthodont. 2004;17(1):35-8.

3. Yüksel E, Zaimoğlu A. Influence of marginal fit and cement types on microleakage of all-ceramic crown systems. Braz Oral Res. 2011;25(3):261-6. doi:10.1590/S1806-83242011000300012

4. Borba M, Miranda WG Jr, Cesar PF, Griggs JA, Bona AD. Evaluation of the adaptation of zirconia-based fixed partial dentures using micro-CT technology. Braz Oral Res. 2013;27(5):396-402. doi:10.1590/S1806-83242013000500003

5. Fasbinder DJ. Computerized technology for restorative dentistry. Am J Dent. 2013;26(3):115-20.

6. Wassell RW, Barker D, Walls AWG. Crowns and other extracoronal restorations: impression materials and technique. $\mathrm{Br}$ Dent J. 2002;192(12):679-90. doi:10.1038/sj.bdj.4801456

7. Al-Bakri IA, Hussey D, Al-Omari WM. The dimensional accuracy of four impression techniques with the use of addition silicone impression materials. J Clin Dent. 2007;18(2):29-33.

8. Giordano R, 2nd. Impression materials: basic properties. Gen Dent. 2000;48(5):510-6.

9. Hamalian TA, Nasr E, Chidiac JJ. Impression materials in fixed prosthodontics: influence of choice on clinical procedure. J Prosthodont. 2011;20(2):153-60. doi:10.1111/j.1532-849X.2010.00673.x

10. Christensen GJ. Impressions are changing: deciding on conventional, digital or digital plus in-office milling. J Am Dent Assoc. 2009;140(10):1301-4. doi:10.14219/jada.archive.2009.0054

11. Boeddinghaus M, Breloer ES, Rehmann P, Wöstmann B. Accuracy of single-tooth restorations based on intraoral digital and conventional impressions in patients. Clin Oral Investig. 2015;19(8):2027-34. doi:10.1007/s00784-015-1430-7

12. Costa JB, Pelogia F, Hagedorn B, Ferracane JL. Evaluation of different methods of optical impression making on the marginal gap of onlays created with CEREC 3D. Oper Dent. 2010;35(3):324-9. doi:10.2341/09-178-L

13. Parsell DE, Anderson BC, Livingston HM, Rudd JI, Tankersley JD. Effect of camera angulation on adaptation of CAD/CAM restorations. J Esthet Dent. 2000;12(2):78-84. doi:10.1111/j.1708-8240.2000.tb00204.x

\section{Acknowledgements}

The authors wish to thank the Neodent Company (Curitiba, Brazil) for the use of the scanners and fabrication of the zirconia copings.

14. Silva JSA, Erdelt K, Edelhoff D, Araujo E, Stimmelmayr $\mathrm{M}$, Vieira LC et al. Marginal and internal fit of four-unit zirconia fixed dental prostheses based on digital and conventional impression techniques. Clin Oral Investig. 2014;18(2):515-23. doi:10.1007/s00784-013-0987-2

15. Ender A, Mehl A. Full arch scans: conventional versus digital impressions: an in-vitro study. Int J Comput Dent. 2011;14(1):11-21.

16. Seelbach P, Brueckel C, Wöstmann B. Accuracy of digital and conventional impression techniques and workflow. Clin Oral Investig. 2013;17(7):1759-64. doi:10.1007/s00784-012-0864-4

17. Laurent M, Scheer P, Dejou J, Laborde G. Clinical evaluation of the marginal fit of cast crowns: validation of the silicone replica method. J Oral Rehabil. 2008;35(2):116-22. doi:10.1111/j.1365-2842.2003.01203.x

18. Boening KW, Wolf BH, Schmidt AE, Kästner K, Walter MH. Clinical fit of Procera AllCeram crowns. J Prosthet Dent. 2000;84(4):419-24. doi:/10.1067/mpr.2000.109125

19. Syrek A, Reich G, Ranftl D, Klein C, Cerny B, Brodesser J. Clinical evaluation of all-ceramic crowns fabricated from intraoral digital impressions based on the principle of active wavefront sampling. J Dent. 2010;38(7):553-9. doi:10.1016/j.jdent.2010.03.015

20. Chaffee NR, Bailey JH, Sherrard DJ. Dimensional accuracy of improved dental stone and epoxy resin die materials. Part I: single die. J Prosthet Dent. 1997;77(2):131-5.

21. Chen SY, Liang WM, Chen FN. Factors affecting the accuracy of elastometric impression materials. J Dent. 2004;32(8):603-9. doi:10.1016/j.jdent.2004.04.002

22. Fransson B, Oilo G, Gjeitanger R. The fit of metal-ceramic crowns, a clinical study. Dent Mater. 1985;1(5):197-9. doi:10.1016/S0109-5641(85)80019-1

23. Nam SJ, Yoon MJ, Kim WH, Ryu GJ, Bang MK, Huh JB. Marginal and internal fit of conventional metal-ceramic and lithium disilicate CAD/CAM crowns. Int J Prosthodont. 2015;28(5):519-21. doi:10.11607/ijp.4089

24. McLean JW, von Fraunhofer JA. The estimation of cement film thickness by an in vivo technique. Br Dent J. 1971;131(3):107-11. doi:10.1038/sj.bdj.4802708

25. Anadioti E, Aquilino SA, Gratton DG, Holloway JA, Denry IL, Thomas GW et al. Internal fit of pressed and computer-aided design/computer-aided manufacturing ceramic crowns made from digital and conventional impressions. J Prosthet Dent. 2015;113(4):304-9. doi:10.1016/j.prosdent.2014.09.015 
26. Pradíes G, Zarauz C, Valverde A, Ferreiroa A, Martinez-Rus F. Clinical evaluation comparing the fit of all-ceramic crowns obtained from silicone and digital intraoral impressions based on wavefront sampling technology. J Dent. 2015;43(2):201-8. doi:10.1016/j.jdent.2014.12.007

27. Reich S, Uhlen S, Gozdowski S, Lohbauer U. Measurement of cement thickness under lithium disilicate crowns using an impression material technique. Clin Oral Investig. 2011;15(4):521-6. doi:10.1007/s00784-010-0414-x
28. Pfeiffer J. Dental CAD/CAM technologies: the optical impression (II). Int J Comput Dent. 1999;2(1):65-72.

29. Flugge TV, Schlager S, Nelson K, Nahles S, Metzger MC. Precision of intraoral digital dental impressions with iTero and extraoral digitization with the iTero and a model scanner. Am J Orthod Dentofacial Orthop. 2013;144(3):471-8. doi:10.1016/j.ajodo.2013.04.017 